\title{
Transparency of Haptic Telepresence Systems with Constant Time Delay
}

\author{
Sandra Hirche, Andrea Bauer, and Martin Buss
}

\begin{abstract}
This paper investigates how the network induced delay together with stabilizing strategies affect the performance of haptic telepresence systems in terms of transparency (human operators should feel as if they were directly acting in the remote environment). Therefore, the mechanical impedance (force over velocity) perceived by the human operator is compared with the real environment impedance in terms of their physical parameters stiffness, damping and mass dependent on the delay. The results are discussed from the human haptic perception point of view and validated in a one-degree-offreedom telepresence experiment.
\end{abstract}

\section{INTRODUCTION}

In a multimodal telepresence system a human operator commands a remote robot (teleoperator) by manipulating the human system interface (HSI). Sensors at the telerobot measure environment interaction, which are then communicated and fed back to the human operator using the corresponding multimodal HSIs, see Fig. 1. Application areas of this technology reach from tele-surgery, -maintenance to teletraining and -entertainment. The focus of this paper is on the haptic (force) feedback system forming a closed-loop control system over a communication network, e.g. the Internet, the human operator and the generally unknown remote environment. The closed loop is affected with unpredictable time delay and packet loss.

In order to guarantee stability with time delay the passivity concept using the scattering transformation [1], equivalently the wave variable transformation [2], is employed, where velocity and force signals are exchanged between the HSI and the teleoperator. Recently, passivity based control methods have been developed to cope with the effects of time-varying delay and packet loss [3].

Transparency - in the sense that the technical systems and communication network should not be felt by the human operator, i.e. the operator should feel as if directly being present and active in the remote environment - is one of the key challenges in telepresence systems. Perfect transparency from the engineering point of view is difficult to achieve in real systems. However, such a technically non-transparent system may still appear transparent to the human due to human haptic perception limits. Hence, the consideration of human haptic perception is an important issue for the analysis and synthesis of networked telepresence system control architectures.

The interesting question is how communication network parameters and the control architecture influence the trans-

All authors' affiliation: Institute of Automatic Control Engineering, Technische Universität München, D-80290 München, Germany http: //www.lsr.ei.tum.de,

S.Hirche@ieee.org, Andrea@mytum.de, M.Buss@ieee.org

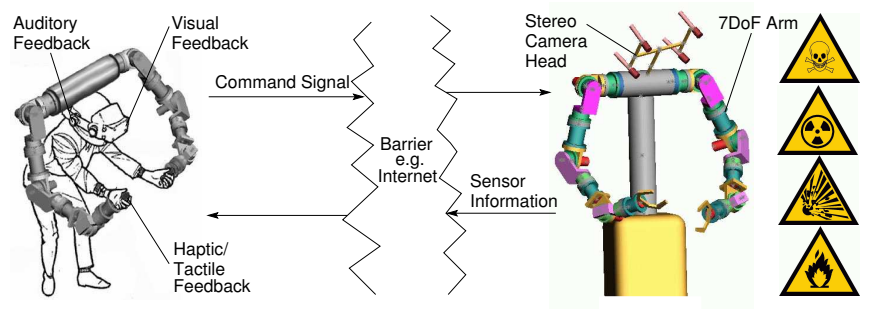

Fig. 1. Multimodal telepresence system.

parency of the haptic telepresence system determining the range of possible applications. The transparency criteria known from literature [4], [5] turn out to be too strict in general considering psychophysical studies of human haptic perception [6] and difficult (if not impossible) to satisfy for communication time delay.

As a first step towards a human perception oriented transparency evaluation for haptic telepresence over packet switched networks this paper contributes a method for transparency analysis in the presence of constant delay. The results and their implications are discussed from a human perception point of view.

The remainder of this paper is organized as follows: Section 2 introduces passivity based stabilization methods and transparency measures; Section 3 presents tranparency analysis methods, discusses the influence of communication network parameters on the impedance distortions (differences of the perceived compared to the true environment impedance) in human perception; section 4 presents experimental results.

\section{TheORETICAL BACKGROUND}

The haptic telepresence system basically consists of a force feedback capable HSI (variables indexed ${ }_{h}$ ) and the teleoperator (index ${ }_{t}$ ) interacting with an usually unknown remote environment (index $e$ ) as shown in Fig. 2. In bilateral telepresence the human manipulates the HSI applying the force $f_{h}$. Based on stability arguments in the standard architecture the HSI velocity $\dot{x}_{h}$ is communicated to the teleoperator where the local velocity control loop ensures the tracking of the desired teleoperator velocity $\dot{x}_{t}^{d}\left({ }^{d}\right.$ denotes desired). The force $f_{e}$ sensed at the remote site, resulting from the interaction with the environment, is transmitted back to the HSI serving as reference signal $f_{h}^{d}$ for the local force control. The time delays $T_{1}, T_{2}$ in the forward and backward path, respectively, see Fig. 2, are assumed to be constant. Without further control measures the system is unstable due to the time delay. 


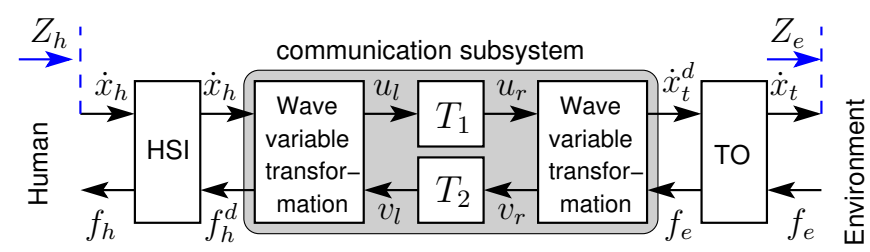

Fig. 2. Telepresence system architecture with wave variable transformation.

\section{A. Stability by Passivity Approach}

The passivity concept provides a sufficient condition for stability of the haptic feedback system. A complex system of interconnected network elements ( $n$-ports) is passive if each of the subsystems is passive. A passive element is one for which, given zero energy storage at $t=0$, the property

$$
\int_{0}^{t} P_{\text {in }}(\tau) \mathrm{d} \tau=\int_{0}^{t} u^{T}(\tau) y(\tau) \mathrm{d} \tau \geq 0 \quad \forall t>0
$$

holds, with $P_{i n}(\tau)$ denoting the power input to the system, $u(\tau), y(\tau)$ the input and output vector. In classical teleoperation architectures, as proposed in [1], the appropriately locally controlled HSI and teleoperator exchange velocity and force signals. As a result the teleoperator/environment and the human/HSI are considered passive subsystems. The wave variable transformation [1], [2] passifies the communication two-port for constant delays with the transformation equations

$$
\begin{aligned}
& u_{l}=\frac{1}{\sqrt{2 b}}\left(f_{h}^{d}+b \dot{x}_{h}\right) ; \quad u_{r}=\frac{1}{\sqrt{2 b}}\left(f_{e}+b \dot{x}_{t}^{d}\right) ; \\
& v_{l}=\frac{1}{\sqrt{2 b}}\left(f_{h}^{d}-b \dot{x}_{h}\right) ; \quad v_{r}=\frac{1}{\sqrt{2 b}}\left(f_{e}-b \dot{x}_{t}^{d}\right) .
\end{aligned}
$$

The tuning parameter $b$ represents the wave impedance of the communication line.

\section{B. Transparency and Human Haptic Perception}

The design goal of the haptic feedback system is that the human operator cannot distinguish between direct interaction with an environment and teleoperated interaction with an remote environment. Then the system is called transparent. In order to evaluate the transparency commonly objective performance metrics are employed. For transparency the position and force at the HSI and the teleoperator are required to be equal in [5]; according to [4] transparency requires the equality of the impedance perceived by the human and the environment impedance

$$
Z_{h}=Z_{e},
$$

with the mechanical impedance $Z$ defined as the mapping from velocity $\dot{x}$ to force $f$. In most cases the considered impedances can sufficiently well approximated by a LTI system; then the impedance can be represented by the transfer function $Z(s)=f(s) / \dot{x}(s)$.

The transparency requirements are difficult to satisfy in a real system, especially with time delay. On the other hand, the knowledge of psychophysical effects in human haptic perception is not incorporated, i.e. the transparency requirements are overly strict in general.
According to numerous psychophysical studies the human being is only able to discriminate velocity and force changes which have a magnitude proportional to the signal value itself. The detection threshold, called just noticable difference (JND), for force perception with hand and arm is around 10\% [6], for velocity around 8\% [7]. Similar detection thresholds exist for the mechanical parameters such as inertia, damping and stiffness. The JND for stiffness perception, for instance, with fingers is around 8\% [8], with hand and arm (cross-limb) 23\% [9]. These results encourage the transparency analysis based on the mechanical parameters of the perceived impedance.

\section{TRANSPARENCY ANALYSIS}

In order to evaluate the influence of the communication delay on the perceived mechanical properties the perceived impedance $Z_{h}$ is computed with the reformulated equations (2)

$$
Z_{h}(s)=b \frac{1+R e^{-s T}}{1-R e^{-s T}} \quad \text { with } \quad R=\frac{Z_{e}-b}{Z_{e}+b} .
$$

and the round-trip delay $T=T_{1}+T_{2}$. For vanishing delay $T=0$ the perceived impedance is equal to the environment impedance. As the influence of the time delay is the major issue in this paper the dynamics of the teleoperator and the HSI are neglected for this analysis. The teleoperator/environment impedance is considered equal to the environment impedance $Z_{e}$, the impedance displayed to the HSI equal to the impedance $Z_{h}$ perceived by the human.

The main challenge for an intuitive physical interpretation of the perceived impedance is the complexity of its transfer function (3). Due to the delay element this transfer function has an infinite number of poles and zeros. Therefore the perceived impedance is approximated by a lower order system.

\section{A. Analytical Low Frequency Approximation}

The approximation of the perceived impedance transfer function is computed using a Padé series of finite order to approximate the delay transfer functions in (3). The order of the perceived impedance approximation depends on the order $N$ of the Padé approximation. In order to simplify the analysis the delay elements are approximated by a first order Padé series

$$
e^{-s T} \approx \frac{1-\frac{T}{2} s}{1+\frac{T}{2} s} .
$$

Generally, a Padé approximation of order $N$ is valid for frequencies $\omega<N /(3 T)$, consequently, the first order approximation is valid for frequencies $\omega<1 /(3 T)$. Inserting (4) in (3) the approximated perceived impedance is

$$
Z_{h}(s) \approx Z_{h}^{\mathrm{app}}(s)=b \frac{2 Z_{e}+b T s}{2 b+T Z_{e} s}
$$

In accordance to the limited frequency range of approximation for further analysis this transfer function is split 
into a low frequency component $Z_{h, l f}^{\text {app }}$ and a high frequency component $F_{h f}$ in series connection

$$
Z_{h}^{\mathrm{app}}(s)=Z_{h, l f}^{\mathrm{app}}(s) F_{h f}(s)
$$

with the high frequency component having approximatly unity gain at lower frequencies

$$
\left|F_{h f}(s)\right| \approx 1 \quad \text { for } \quad \omega<\frac{1}{3 T} .
$$

The component $Z_{h, l f}^{\text {app }}$ represents a good approximation of the low frequency behavior of the perceived impedance. Generally, only lower frequencies are of interest as the bandwidth of human haptic (proprioreceptive and kinesthetic) perception is limited to app. $60 \mathrm{~Hz}$. The mechanical parameters of the approximated perceived impedance $Z_{h, l f}^{\text {app }}$ can be derived analytically as a function of the round-trip time delay $T$ and the wave impedance $b$. A transparency analysis based on the proposed approach is exemplarily carried out in detail for the prototypical cases free space motion and contact with a stiff wall in the following.

\section{B. Approximated Perceived Impedance for Prototypical En- vironment Impedances}

1) Free Space Motion: In free space motion no environment force is exerted on the teleoperator $f_{e}=0$, i.e. the environment impedance is $Z_{e}=0$. The exact perceived impedance (3) is

$$
Z_{h}(s)=b \frac{1-e^{-s T}}{1+e^{-s T}} .
$$

Inserting the environment impedance into (5) gives the approximation of the perceived impedance valid for low frequencies

$$
Z_{h}^{\text {app }}(s)=m_{h} s \frac{1}{\frac{T}{2} s+1} .
$$

with

$$
m_{h}=\frac{b T}{2} \text {. }
$$

The lefthand factor in (8) represents the low frequency component $Z_{h, l f}^{\text {app }}$ in (6). The righthand factor is the high frequency component $F_{h f}$ satisfying (7), in fact, in steady state $\left|F_{h f}(0)=1\right|$ holds. The similarity of the exact and the approximated perceived impedance for low frequencies can also be observed from their frequency responses for a simulated example depicted in Fig. 3. Thus, at low frequencies the perceived impedance is an inertia with the mass $m_{h}(9)$.

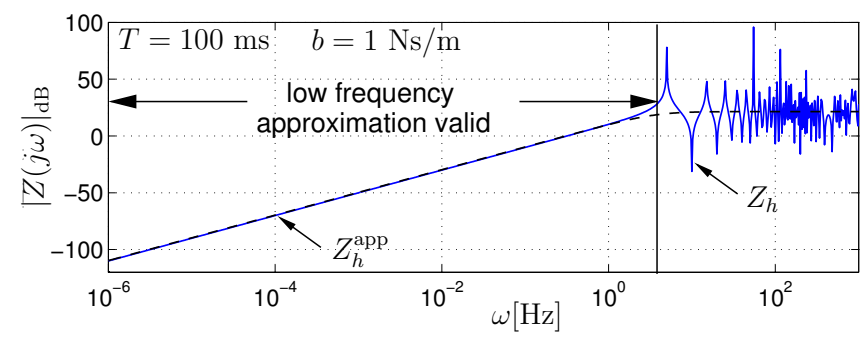

Fig. 3. Amplitude/frequency characteristics of the perceived and the approximated impedance in free space motion.

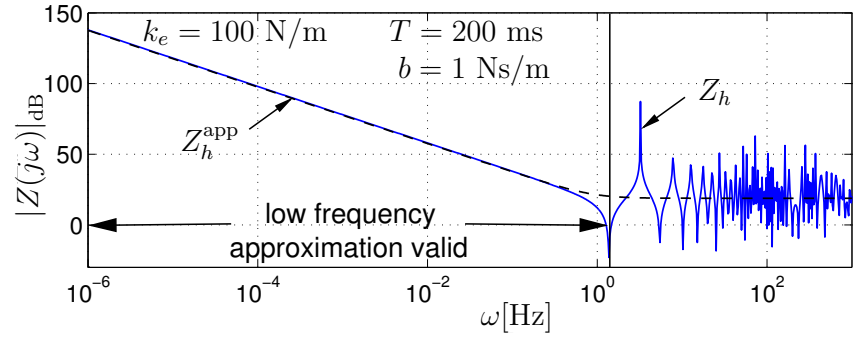

Fig. 4. Amplitude/frequency characteristics of the perceived and the approximated impedance in contact.

2) Contact with a Stiff Wall Environment: In contact with a stiff wall a force proportional with the stiffness coefficient $k_{e}$ to the wall penetration depth acts on the teleoperator, i.e. the environment impedance is described by the transfer function $Z_{e}=k_{e} / s$. The exact perceived impedance (3) is computed by

$$
Z_{h}(s)=b \frac{k_{e}+b s+\left(k_{e}-b s\right) e^{-s T}}{k_{e}+b s-\left(k_{e}-b s\right) e^{-s T}} .
$$

The approximation (5) of the perceived impedance for low frequency is analogously computed to the free space motion case

$$
Z_{h}^{\text {app }}(s)=\frac{k_{h}}{s}\left(1+\frac{b T}{2 k_{e}} s^{2}\right)
$$

with

$$
k_{h}=\frac{2 b k_{e}}{2 b+T k_{e}} .
$$

The lefthand factor is the low frequency component $Z_{h, l f}^{\text {app }}$ from (6). The right hand factor in (10) exhibits high pass behavior satisfying (7), in fact, in steady state as in the free space motion case $\left|F_{h f}(0)=1\right|$ holds. The frequency responses for the exact and the approximated perceived impedance shown in Fig. 4 are similar for low frequencies, hence validate the approach.. The integrating characteristics $Z_{h, l f}^{\text {app }}=k_{h} / s$ can be taken as a good approximation of the low frequency behavior. As a result the perceived impedance in contact at low frequency has a springlike behavior but with a lower stiffness $k_{h}$ than the environment stiffness $k_{e}$. In fact, the communication subsystem including the wave variable transformation can be interpreted as a rod with a stiffness coefficient $2 b / T$ in mechanical series connection with the environment.

\section{TRANSPARENCY INSIGHTS}

The objective and human perception oriented interpretation of the obtained results leads to the following insights.

\section{A. Communication Induced Inertia Perception}

In free space motion an inertia is perceived even though no inertia is contained in the environment. The inertia characteristics is induced by the wave variable transformation and the communication delay. The inertia mainly depends on the round-trip delay $T$ and the wave impedance $b$ (9). 


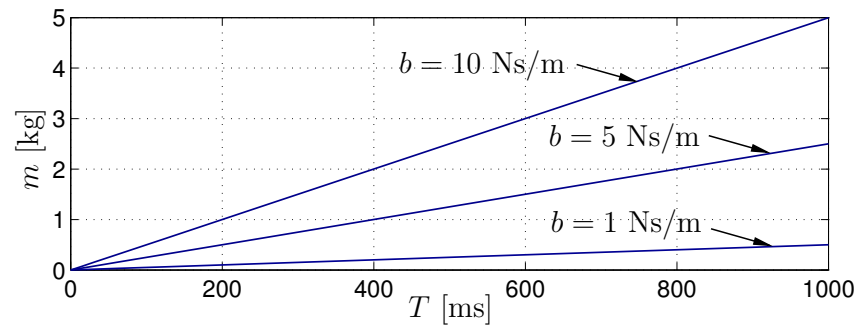

Fig. 5. Perceived inertia in free space motion depending on round-trip time delay.

With increasing round-trip delay the inertia proportionally grows as shown in Fig. 5. The perceived inertia also increases with the wave impedance. For large time delay, environments without spring characteristics are transparent only if the wave impedance is tuned to a very small value.

\section{B. Communication Induced Stiffness Reduction}

If the environment exhibits spring characteristics the operator perceives a substantially reduced stiffness. The environment feels softer than it really is. The perceived stiffness coefficient (11) depends on the the communication delay as shown in Fig. 6, and the environment stiffness. The reduction of the perceived stiffness becomes percentually higher for high environment stiffness. High values of the wave impedance $b$ increase the transparency of stiff environments. However, this contradicts the design rule for the wave impedance in environments without spring characteristics.

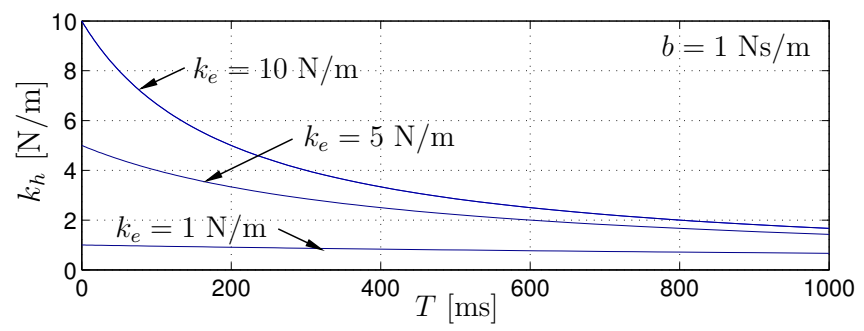

Fig. 6. Perceived stiffness in contact depending on round-trip time delay for different environment stiffness coefficients.

\section{Communication Induced Stiffness Bound}

The perceived stiffness (11) can never exceed

$$
k_{h, \max }=\lim _{k_{e} \rightarrow \infty} k_{h}=\frac{2 b}{T} .
$$

This result is indicated in Fig. 7 by the asymptotic behavior of the perceived stiffness for increasing environment stiffness. Considering the psychophysical fact that the human feels a wall to be rigid for $k_{h} \geq 24200 \mathrm{~N} / \mathrm{m}$ [10] it becomes clear that only for a very small time delay and a very large wave impedance $b$ a rigid wall can be realistically displayed with this control architecture. For large time delay the stiffness, especially in case of hard walls, is not transparent. Appropriate tuning (high values) of the wave impedance $b$ increases the transparency in terms of the maximum displayable stiffness.

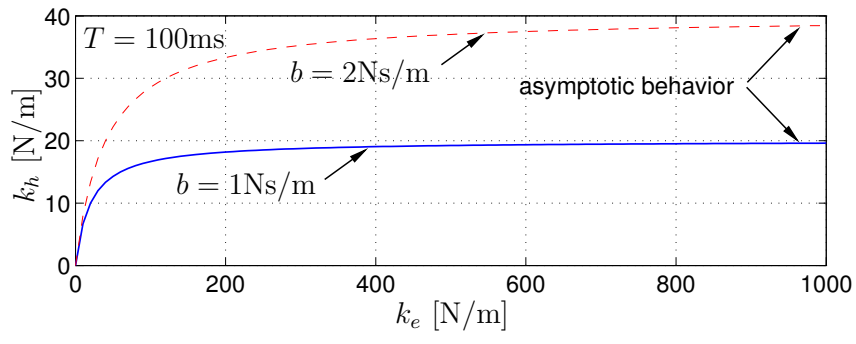

Fig. 7. Perceived stiffness depending on environment stiffness.

\section{Bounded Perceivable Stiffness Change}

In some tasks not only the absolute value of the perceived stiffness is important but also the possibility to distinguish between differently stiff environments. This is especially important for soft environments (e.g. in tele-surgery), where different characteristics have to be distinguished.

As indicated by the asymptotic behavior of the perceived stiffness in Fig. 7 at higher values of the environment stiffness any stiffness change in the environment results in a very small change in the perceived stiffness. According to the human haptic perception characteristics, see Section II-B, a change in the environment stiffness from a reference value $k_{e}^{0}$ to the value $k_{e}$ is perceivable by the human only if the corresponding percentual change in the perceived stiffness

$$
\delta k_{h}=\left|k_{h}-k_{h}^{0}\right| / k_{h}^{0}
$$

is larger than the stiffness JND, denoted by $\mathrm{JND}_{k}$

$$
\delta k_{h}=\frac{2 b \delta k_{e}}{2 b+T k_{e}} \geq \mathrm{JND}_{k}
$$

with the percentual change in the environment stiffness $\delta k_{e}$ defined analogously to (13) and the perceived reference stiffness coefficient $k_{h}^{0}=k_{h}\left(k_{e}^{0}\right)$ according to (11). The percentual change of perceived stiffness $\delta k_{h}$ and environment stiffness $\delta k_{e}$ is equal only for the marginal cases of zero delay or infinite wave impedance. At high delay and high environment stiffness a large change in the environment stiffness may result in a non-perceivable change of the perceived stiffness. In fact, for a given delay and wave impedance there exists an upper bound in the environment stiffness $k_{e, \max }$ at which a human is no longer able to detect the change to a very large (mathematically infinite) value of environment stiffness with $k_{e, \max }=k_{e}\left([1-J N D] k_{h, \max }\right)$ (12). This maximum allowable environment stiffness depending on the time delay is depicted in Fig. 8 for the two different JND assumptions reported in the literature (23\% in [9], $8 \%$ in [8]). According to (14) the appropriate tuning (high values) of the wave impedance increases the transparency in terms of the range of environment stiffness where a change can be perceived.

\section{E. Just Noticeable Time Delay Difference}

Based on the assumption that a delay difference is haptically perceived only by the difference in the mechanical properties of the perceived impedance it is conjectured that the JND for the time delay can be computed from the 


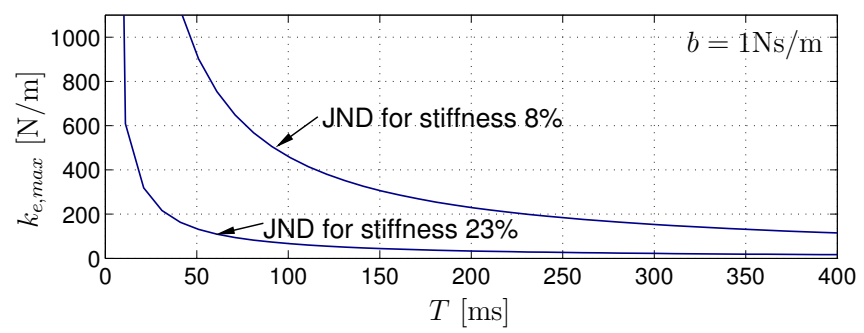

Fig. 8. Maximum environment stiffness at which a change to infinite environment stiffness is still perceivable by the human depending on roundtrip time delay.

well-known JND's for mechanical properties. This result is interesting with respect to the design of control architectures for telepresence sytems over the Internet, coping with packet loss and time-varying delay, where data buffering strategies, as e.g. in [3], introduce additional delay. If the additional delay is below the human perception threshold then no change in transparency should be perceived.

The percentual difference of the perceived inertia $\delta m_{h}$ and the perceived stiffness $\delta k_{h}$, both defined analogously to (13), are considered. The reference values in (13) for the inertia $m_{h}^{0}=m_{h}\left(T^{0}\right)$ (9) and the stiffness $k_{h}^{0}=k_{h}\left(T^{0}\right)(11)$ represent the perceived mechanical properties at the reference delay $T^{0}$.

An absolute time delay difference $\Delta T=\left|T-T^{0}\right|$ is perceivable by the human if the corresponding percentual difference of the perceived mechanical property is larger or equal to the corresponding JND. Accordingly, for free space motion

$$
\delta m_{h} \geq \mathrm{JND}_{m}
$$

must hold with $\mathrm{JND}_{m}$ denoting the inertia JND, and for contact with a stiff wall

$$
\delta k_{h} \geq \mathrm{JND}_{k} .
$$

Inserting (9) in (15) and (11) in (16) gives the time delay JND for free space motion and contact, respectively. According to that a delay difference is expected to be perceivable by the human in free space motion if

$$
\Delta T \geq \mathrm{JND}_{m} T^{0} .
$$

Due to linear dependence of the perceived inertia in free space motion the time delay JND can be defined as a percentual JND which is equal to the inertia JND. In contact with a stiff wall a delay difference is perceivable by the human if

$$
\Delta T \geq \frac{\mathrm{JND}_{k}}{\mathrm{JND}_{k}+1}\left(\frac{2 b}{k_{e}}+T^{0}\right) .
$$

In contrast to the free space motion case the just perceivable time delay difference here depends on the environment stiffness $k_{e}$, the wave impedance $b$ and the reference time delay $T^{0}$. The detection threshold $\Delta T$ becomes smaller with decreasing reference time delay. Consequently, at low reference time delay any additional delay degrades the transparency more than at high reference time delay.

\section{EXPERIMENTS}

In the first experiment the theoretically obtained dependency of the perceived impedance parameters on the roundtrip time delay is validated. In the second one a preliminary human user study is conducted in order to give a clue on the how time delay degrades the human perceived transparency. The just noticeable time delay difference is determined. In both experiments, the prototypical cases of free space motion with zero environment force and contact with a stiff wall (stiffness coefficient $k_{e}=12500 \mathrm{~N} / \mathrm{m}$ ) are investigated.

\section{A. Experimental Setup}

The experimental setup, see Fig. 9, consists of a single degree-of-freedom force feedback paddle, refer to [11] for more details, connected to a PC. The paddle DC motor torque is controlled by the PWM amplifier. The force applied to the paddle lever is measured by a strain gauge bridge, the position of the lever by an optic pulse incremental encoder. A virtual environment is used instead of a real teleoperator/environment in order to seperately consider the prototypical environment scenarios. The virtual environment, the control loops, the model of the communication subsystem with different constant delay and the wave variable transformation with a wave impedance $b=125 \mathrm{Ns} / \mathrm{m}$ are composed of MATLAB/SIMULINK blocksets; standalone realtime code for RT Linux is automatically generated from that. All experiments were performed with a sample time interval $T_{A}=0.001 \mathrm{~s}$.

\section{B. Objective Measurements}

The perceived inertia $m_{h}$ in free space motion and the perceived stiffness coefficient $k_{h}$ in contact with the wall are determined depending on the round-trip delay that is varied within the interval $T \in[5,400] \mathrm{ms}$. The parameters $m_{h}$ and $k_{h}$ are determined by a least squares identification from the measured HSI position and HSI force signals. The results for the perceived inertia in free space motion are shown in Fig. 10 (a), and for the perceived stiffness in contact in Fig. 10 (b). The theoretically obtained dependencies of these parameters on the round-trip delay given by (9) and (11) are convincingly validated.

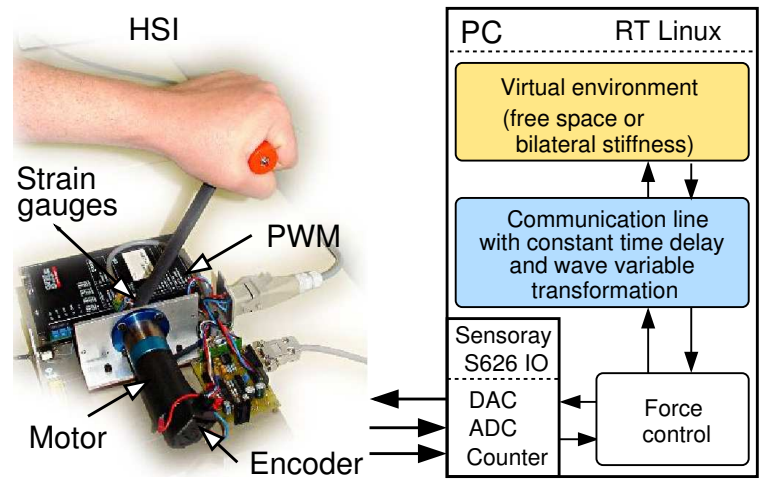

Fig. 9. Experimental system architecture. 


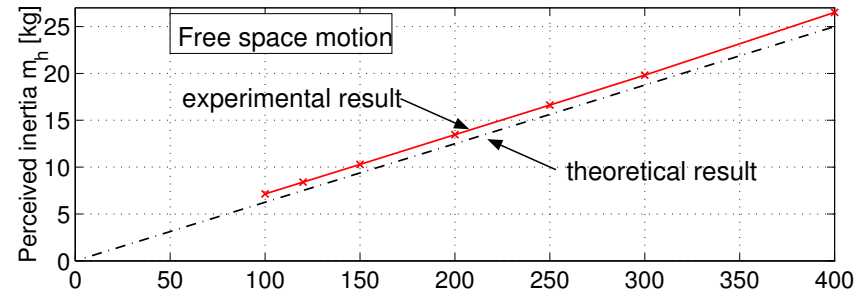

(a)

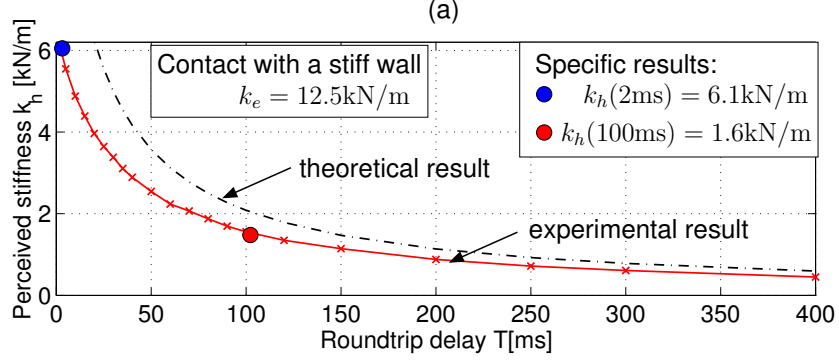

(b)

Fig. 10. Experimentally obtained perceived inertia $m_{h}$ (a) and stiffness $k_{h}$ depending on round-trip delay $T$ compared to theoretical results.

\section{Human User Study}

Four experiments with 7 test persons are performed for two different reference round-trip delays $T^{0}=2 \mathrm{~ms}$ and $T^{0}=100 \mathrm{~ms}$ for each of the considered prototypical cases 'free space motion' and 'contact with a wall' using the same parameters as in the foregoing experiment. Therefore the well-known three interval forced choice (3IFC) paradigm is applied which is a common experimental tool in psychophysics to determine detection thresholds in human haptic perception [12]. The main feature is that the subjects are presented three consecutive time intervals, 20 s each, two with the reference value $T^{0}$ of the time delay, one with a different time delay value $T$. The subject has to tell which of the intervals felt different. Starting from a non-perceivable delay difference $\Delta T$ this value is increased after every incorrect answer until three consecutive correct answers on the same value $\Delta T$ are given. Three of these passes are performed, the mean value over the passes is considered the subject specific detection threshold.

The results for all four experiments are shown in Fig. 11, where $\overline{\Delta T}$ denotes the average over all subjects. As expected from the theoretical results in Section IV-E, in both scenarios the average detected delay difference is smaller for low reference time delay. The Student's test is performed giving a statement about the statistical significance of the detection thresholds difference $\Delta T_{2 \mathrm{~ms}}-\Delta T_{100 \mathrm{~ms}}$. For 'contact with the wall' the mean detection threshold for low reference delay is statistically significant (95\%) smaller than for high reference delay $\overline{\Delta T}_{2 \mathrm{~ms}}<\overline{\Delta T}_{100 \mathrm{~ms}}$. For 'free space motion' it is not significant $(90 \%)$ in a statistical sense. A more significant result is to be expected for more test persons.

The results of this preliminary human user study validate the conjecture from Section IV-E, hence the proposed analysis of the transparency degradation by time delay over known haptic perception limits. Further psychophysical experiments are necessary to confirm this conclusion.

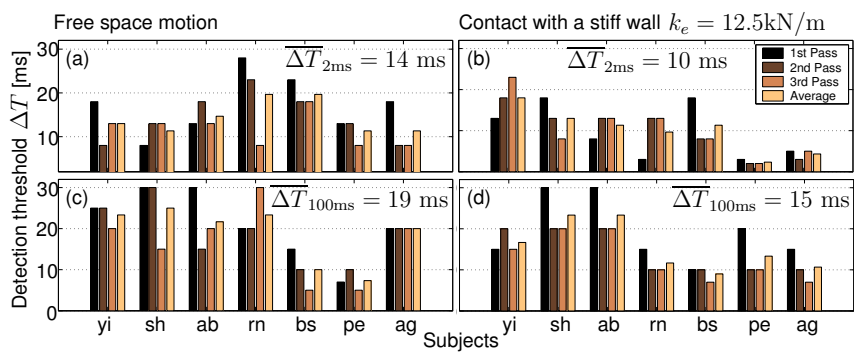

Fig. 11. Results of human user study: detection thresholds for time delay $\Delta T$ at reference time delays $T_{0}=2 \mathrm{~ms}$ (a), (b) and $T_{0}=100 \mathrm{~ms}$ (c), (d) for free space motion (a), (c) and contact with a stiff wall (b), (d).

\section{CONCLUSIONS}

In this paper a method for the transparency analysis of haptic (force feedback) telepresence systems is presented with the goal to quantify the degradation induced by communication effects from a human perception point of view. Therefore the effect of the communication time delay and the wave variable transformation on the mechanical properties of the impedance perceived by the human is analyzed. The interpretation of the results using known psychophysical facts reveals interesting insights with implications for the control design and the range of tele-applications depending on the communication time delay. The prototypical scenarios of the teleoperator in free motion and in contact with stiff environments are investigated theoretically and experimentally, both in objective experiments and human user studies.

\section{REFERENCES}

[1] R. Anderson and M. Spong, "Bilateral Control of Teleoperators with Time Delay," IEEE Transactions on Automatic Control, vol. 34, no. 5, pp. 494-501, 1989.

[2] G. Niemeyer and J.-J. Slotine, "Stable Adaptive Teleoperation," IEEE Journal of Oceanic Engineering, vol. 16, no. 1, pp. 152-162, 1991.

[3] B. Berestesky, N. Chopra, and M. W. Spong, "Discrete Time Passivity in Bilateral Teleoperation over the Internet," in Proceedings of the IEEE International Conference on Robotics and Automation ICRA'04, New Orleans, US, 2004, pp. 4557-4564.

[4] D. Lawrence, "Stability and Transparency in Bilateral Teleoperation," IEEE Transactions on Robotics and Automation, vol. 9, no. 5, pp. 624-637, October 1993.

[5] Y. Yokokohji and T. Yoshikawa, "Bilateral Control of Master-Slave Manipulators for Ideal Kinesthetic Coupling Formulation and Experiment," IEEE Transactions on Robotics and Automation, vol. 10, no. 5, pp. 605-619, October 1994.

[6] G. Burdea, Force and Touch Feedback for Virtual Reality. John Wiley, 1996.

[7] L. A. Jones and I. W. Hunter, "Human Operator Perception of Mechanical Variables and Their Effects on Tracking Performance," ASME Advances in Robotics, vol. 42, pp. 49-53, 1992.

[8] D. N. B. G. Tan, H. Z. and M. A. Srinivasan, "Manual Discrimination of Compliance Using Active Pinch Grasp: The Role of Force and Work Cues," Perception and Psychophysics, vol. 57, pp. 495-510, 1995.

[9] L. Jones and I. Hunter, "A Perceptual Analysis of Stiffness," Experimental Brain Research, vol. 79, pp. 150-156, 1990.

[10] S. M. A. E. B. Tan, H. Z. and B. Cheng, "Human Factors for the Design of Force-Reflecting Haptic Interfaces," ASME Dynamic Systems and Control Division, vol. 1, pp. 353-359, 1994.

[11] H. Baier, M. Buss, and G. Schmidt, "Control Mode Switching for Teledrilling Based on a Hybrid System Model," in Proceedings of the IEEE/ASME International Conference on Advanced Intelligent Mechatronics AIM'97, Tokyo, Japan, Paper No. 50, 1997.

[12] G. Gescheider, Psychophysics: The Fundamentals. Hillsdale: Lawrence Erlbaum and Associates, 3rd Edition. 